Mathematical Research Letters 5, 623-628 (1998)

\title{
DENSITY OF RATIONAL POINTS ON ENRIQUES SURFACES
}

\author{
F.A. Bogomolov and Yu. Tschinkel
}

\section{Introduction}

Let $X$ be an irreducible algebraic variety defined over a number field $K$. We will say that rational points on $X$ are potentially dense if there exists a finite extension $K^{\prime}$ of $K$ such that the set of $K^{\prime}$-rational points is Zariski-dense in $X$. It seems reasonable to ask whether or not rational points are potentially dense if neither $X$ nor its unramified coverings admit a morphism onto a variety of general type. This question has an easy answer if the dimension of $X=1$. Very little is known in higher dimensions. Clearly, potential density holds for unirational varieties and for abelian varieties. In [6] and [3] it is proved that rational points are potentially dense on all Fano threefolds with the possible exception of double covers of $\mathbb{P}^{3}$ ramified in smooth surfaces of degree 6 .

In this paper we will study the question of density under the additional assumption that $X$ admits the structure of an elliptic fibration $\varphi: X \rightarrow B$ over some irreducible normal base $B$. As one of the applications we prove that rational points on Enriques surfaces are potentially dense. An alternative idea to prove density would have been to use the group of automorphisms $A u t(X)$. There exist Enriques surfaces with a finite group $A u t(X)$ (cf. [5, 1]), but even then the automorphism group of the corresponding K3-cover is infinite (this follows from the classification of Enriques surfaces with finite automorphism groups $[10,8])$.

\section{Elliptic fibrations - Generalities}

Let $K$ be a number field and $X$ a smooth projective algebraic surface defined over $K$. We say that $X$ admits a structure of an elliptic fibration if there exists a regular map $\varphi: X \rightarrow B$ onto a smooth (irreducible) curve $B$ whose fibers are connected curves such that the generic fiber is a smooth curve of genus 1 . We denote by $X_{b}$ the fiber over $b \in B$. We will say that $X$ admits a structure of a Jacobian elliptic fibration if there exists a zero section $e: B \rightarrow X$. It is well known (cf. [2] or [7]) that to every elliptic fibration $\varphi: \mathcal{E} \rightarrow B$ one can associate a Jacobian elliptic fibration $\varphi_{\mathcal{J}}: \mathcal{J}=\mathcal{J}(\mathcal{E}) \rightarrow B$ (over the same groundfield), which over the generic point of the base $B$ is given by classes of divisors of degree

Received September 1, 1998, revised October 26, 1998. 
zero in the fiber. The zero section corresponds to the trivial class. There is a fiberwise action of $\mathcal{J}$ on $\mathcal{E}$, more precisely, a rational map

$$
\psi: \mathcal{J} \times{ }_{B} \mathcal{E} \rightarrow \mathcal{E}
$$

which is regular in non-singular points of the fibers of $\mathcal{J}$ and $\mathcal{E}$ and which induces a transitive action of $\mathcal{J}_{b}$ on $\mathcal{E}_{b}$ (for smooth fibers).

2.1. Multisections. Let $\varphi: \mathcal{E} \rightarrow B$ be an elliptic fibration. A multisection $i: \mathcal{M} \hookrightarrow \mathcal{E}$ is an irreducible subvariety of $\mathcal{E}$ such that the map $\varphi \circ i: \mathcal{M} \rightarrow B$ is finite and surjective. We will denote by $d=d(\mathcal{M})$ the degree of this projection.

Definition 2.1. Let $\varphi: \mathcal{E} \rightarrow B$ be an elliptic fibration. A multisection $\mathcal{M}$ is said to be of order $m$ if $m$ is the smallest positive integer such that for any $b \in B$ and any pair of points $p_{b}, p_{b}^{\prime} \in \mathcal{M} \cap \mathcal{E}_{b}$ the image of the zero-cycle $p_{b}-p_{b}^{\prime}$ in $\mathcal{J}_{b}$ is torsion of order $m$.

Note that any section of an elliptic fibration is a multisection of order one.

Let $\Phi_{m} \subset \mathcal{J}$ be the subvariety of $m$-torsion points of $\mathcal{J}$ (i.e., all points $p_{b}$ such that $m \cdot p_{b}=0$ in the group of rational points of the corresponding fiber $\mathcal{J}_{b}$,) which are not contained in the zero section $e(B)$.

Lemma 2.2. If $\mathcal{M} \subset \psi\left(\Phi_{m} \times_{B} \mathcal{M}\right)$ then $\mathcal{M}$ is a multisection of order $m$.

Proof. Tautology.

Definition 2.3. Let $\varphi: \mathcal{E} \rightarrow B$ be an elliptic fibration. $A$ saliently ramified multisection of $\mathcal{E}$ is a multisection $\mathcal{M}$ which is ramified in a point $p_{b}$ which lies in a smooth (elliptic curve) fiber $\mathcal{E}_{b}$ of $\mathcal{E}$.

Proposition 2.4. Let $\mathcal{M}$ be a saliently ramified multisection of $\mathcal{E}$. Then $\mathcal{M}$ is not of order $m$ for any $m>0$.

Proof. By assumption, nearby fibers of $\mathcal{E}_{b}$ are smooth. Consider an embedding $K \hookrightarrow \mathbb{C}$. We can find a sequence $\left(b_{i}\right) \in B(\mathbb{C})$ of points in the base converging to $b$ (in complex topology) and pairs of distinct points $p_{i}, p_{i}^{\prime}$ in the fibers $\mathcal{E}_{b_{i}}(\mathbb{C})$ which converge to $p$. If we assume that the cycle $p_{i}-p_{i}^{\prime}$ is torsion of order $m$ (for some $m \geq 2$ ) in the Jacobian we obtain a contradiction since it converges to $0=e(b)$.

Definition 2.5. Let $\mathcal{M}$ be a multisection of degree $d$ of the elliptic fibration $\varphi: \mathcal{E} \rightarrow B$ and $b \in B$. We denote by $\operatorname{Tr}_{\mathcal{M}}(b)$ the zero-cycle $\mathcal{M} \cap \mathcal{E}_{b}$. Define the class map

$$
\tau_{\mathcal{M}}: \mathcal{E} \rightarrow \mathcal{J}
$$

by the following rule:

$$
\tau_{\mathcal{M}}(p)=\left[d \cdot p-\operatorname{Tr}_{\mathcal{M}}(\varphi(p))\right] \quad \forall p \in \mathcal{E}
$$


Lemma 2.6. Suppose that the multisection $\mathcal{M}$ is of degree $d$ and not of order $d^{\prime}$ with $d^{\prime} \mid d$. Then the map

$$
\tau_{\mathcal{M}}: \mathcal{M} \rightarrow \tau_{\mathcal{M}}(\mathcal{M})
$$

is a birational isomorphism.

Proof. Consider all points $p, p^{\prime} \in \mathcal{E}_{b}$ such that $\tau_{\mathcal{M}}(p)=\tau_{\mathcal{M}}\left(p^{\prime}\right)$. Then the cycle $p-p^{\prime}$ is of order $d^{\prime} \mid d$ in $\mathcal{J}_{b}$. On the other hand, for a given $d^{\prime}$ pairs of distinct points differing by torsion of order $d^{\prime}$ (more precisely, by a translation by $\left.\Phi_{d^{\prime}}\right)$ constitute a Weil divisor in $\mathcal{M}$. Therefore, the map $\tau_{\mathcal{M}}$ is a birational isomorphism.

Proposition 2.7. The map $\tau_{\mathcal{M}}$ is regular outside of singular fibers of $\mathcal{E} \rightarrow B$.

Proof. Evident.

Corollary 2.8. Let $\mathcal{M}$ be a saliently ramified multisection of $\mathcal{E}$. Then $\tau_{\mathcal{M}}(\mathcal{M})$ is a saliently ramified multisection of $\mathcal{J}$.

Proof. The map $\tau_{\mathcal{M}}$ is unramified on the set of non-singular fibers. By lemma 2.6 , it is a birational isomorphism.

Remark 2.9. Let $\mathcal{M} \subset \mathcal{E}$ be a multisection which is not of order $m$ for any $m$. Then $\tau_{\mathcal{M}}(\mathcal{M})$ is not of order $m$ for any $m$.

From now on we will restict to the case of the base $B=\mathbb{P}^{1}$. Merel's theorem implies:

Proposition 2.10. [3] Let $\varphi_{\mathcal{J}}: \mathcal{J} \rightarrow \mathbb{P}^{1}$ be a Jacobian elliptic fibration defined over $K$ with a saliently ramified multisection $\mathcal{M}$. Then for all but finitely many $b \in \varphi_{\mathcal{J}}(\mathcal{M}(K)) \subset \mathbb{P}^{1}(K)$ the fibers $\mathcal{J}_{b}$ have infinitely many rational points.

Theorem 2.11. Let $\varphi: \mathcal{E} \rightarrow \mathbb{P}^{1}$ be an elliptic fibration defined over $K$. Assume that there exists a rational or elliptic saliently ramified multisection $\mathcal{M}$ defined over $K$. Then rational points on $\mathcal{E}$ are potentially dense.

Proof. Extending the groundfield, we can assume that $K$-rational points on $\mathcal{M}$ are Zariski-dense and that $\mathcal{M}$ intersects a smooth fiber $\mathcal{E}_{\varphi(p)}$ with local intersection multiplicity $\geq 2$ at a $K$-rational point $p$. The image $\varphi(\mathcal{M}(K))$ is Zariski-dense in $\mathbb{P}^{1}$. Every fiber $\mathcal{E}_{b}$ for $b \in \varphi(\mathcal{M}(K))$ contains at least one point $p_{b} \in \mathcal{M}(K)$. Therefore, it suffices to show that for almost all $b \in \varphi(\mathcal{M}(K))$ the fiber $\mathcal{J}_{b}$ has a $K$-rational point of infinite order. Then we use the action of the $\mathcal{J}$ on $\mathcal{E}$ to translate $p_{b}$.

By assumption and 2.8 the curve $\tau_{\mathcal{M}}(\mathcal{M})$ is a saliently ramified multisection of $\mathcal{J} \rightarrow \mathbb{P}^{1}$. The point $\tau_{\mathcal{M}}\left(p_{b}\right) \in \tau_{\mathcal{M}}(\mathcal{M})$ is a $K$-rational point of the fiber $\mathcal{J}_{\varphi\left(p_{b}\right)}$. Moreover, it is contained in the saliently ramified multisection $\tau_{\mathcal{M}}(\mathcal{M})$. By theorem 2.10, for all but finitely many fibers $\mathcal{J}_{\varphi\left(p_{b}\right)}$ the point $\tau_{\mathcal{M}}\left(p_{b}\right)$ is a non-torsion point in the group $\mathcal{J}_{\varphi\left(p_{b}\right)}(K)$. This concludes the proof. 
Remark 2.12. An alternative argument avoiding Merel's theorem goes as follows: We can find a base change $\beta: B^{\prime} \rightarrow B$ with the following properties: $\beta$ is étale at $b$ (corresponding to the smooth fiber of $\mathcal{E} \rightarrow B$ where $\mathcal{M}$ is ramified), $\mathcal{M}$ pulls back to a section $\mathcal{M}^{\prime}$ of $\mathcal{E}^{\prime} \rightarrow B^{\prime}$ and $\mathcal{E}^{\prime}$ acquires a zero section $e^{\prime}$ (which is different from $\mathcal{M}^{\prime}$ ). Then $\mathcal{M}^{\prime}$ must be of infinite order in the Mordell-Weil group of $\mathcal{E}^{\prime}$. A specialization argument ( $c f$. [11]) implies that rational points are (potentially) dense on $\mathcal{E}^{\prime}$.

Proposition 2.13. Let $X$ be a smooth algebraic surface defined over a number field $K$ and admitting two different elliptic fibrations $\varphi_{1}: \mathcal{E}_{1} \rightarrow B_{1}$ and $\varphi_{2}$ : $\mathcal{E}_{2} \rightarrow B_{2}$ with $B_{1} \simeq B_{2} \simeq \mathbb{P}^{1}$. Then rational points on $X$ are potentially dense.

Proof. If there is at least one $\mathcal{E}_{b_{2}}$ which is not of order $m$ for any $m$ we are done by 2.9. In view of 2.8, it suffices to consider the case when for all $b_{2} \in B_{2}$ all multiple intersection points of the multisections $\mathcal{E}_{b_{2}}$ with fibers $\mathcal{E}_{b_{1}}$ (for $b_{1} \in B_{1}$ ) are contained in the singular fibers of the fibration $\mathcal{E}_{1} \rightarrow B_{1}$. If a generic $\mathcal{E}_{b_{2}}$ is of some fixed order $m_{0}$ we see that (a cover of) $X$ is dominated (birationally) by a product of two elliptic curves.

\section{Enriques surfaces}

We start with a brief summary of the structure theory of Enriques surfaces (cf. [2], pp. 274-275): Either $X$ is non-special - then it has visibly two distinct elliptic fibrations over $\mathbb{P}^{1}$ - or it admits an elliptic fibration with a 2-section which is a $(-2)$-curve (this case is called special in [2]). In the special case the associated K3 double cover $\pi: Y \rightarrow X$ admits the structure of an elliptic fibration $Y \rightarrow \mathbb{P}^{1}$ with two (non-intersecting) sections (which could differ by torsion). The surface $Y$ is the minimal resolution of a double covering of a quadratic cone $Q \subset \mathbb{P}^{3}$ (given by $z_{0} z_{1}=z_{2}^{2}$ in standard coordinates in $\mathbb{P}^{3}=\left(z_{0}, z_{1}, z_{2}, z_{3}\right)$,) ramified in an intersection of this cone with a quartic hypersurface in $\mathbb{P}^{3}$ not passing through the vertex of this cone. The ramification curve $R$ is reduced, of degree 8 and has at most simple singularities. The fibers of the elliptic fibration correspond to the generators of the cone $Q$ and the two sections are mapped to the vertex of $Q$ (cf. [2], p. 278).

Using this geometric description we are going to exhibit two transversal elliptic fibrations on $X$. An alternative argument, which uses the combinatorics of the Picard lattice, is contained in [4] (Th. 3.4.1, p. 183).

Theorem 3.1. Let $X$ be an Enriques surface over $K$. Then rational points on $X$ are potentially dense.

Proof. In the non-special case we apply 2.13 and we are done.

Now let us consider the special case. Recall that the elliptic fibration $Y \rightarrow \mathbb{P}^{1}$ has two non-intersecting sections $e_{1}, e_{2}$. Their difference is torsion in the Picard group of the generic point of $Y \rightarrow \mathbb{P}^{1}$ if there exists an integer $m>0$ such that $m\left(e_{1}-e_{2}\right)$ can be represented as a sum, with integer coefficients, of components 
of singular fibers of $Y \rightarrow \mathbb{P}^{1}$. If all fibers of $Y \rightarrow \mathbb{P}^{1}$ are irreducible then the difference $e_{1}-e_{2}$ is not torsion and rational points are potentially dense. Otherwise, we have to consider subcases of the special case.

After blowing up the vertex of the cone we can realize $Q$ birationally as $\mathbb{P}_{\mathbb{P}^{1}}(\mathcal{O}(2) \oplus \mathcal{O})$ (see [3]). Taking sections of $\mathbb{P}_{\mathbb{P}^{1}}(\mathcal{O}(2))$ (a 3-dimensional linear space) we obtain a family (of conics) $\mathbb{P}_{s}^{1} \simeq \mathbb{P}^{1} \subset Q$ where each $\mathbb{P}_{s}^{1}$ intersects the ramification curve $R$ in 8 points (counted with multiplicities). The double cover $D_{s}$ of $\mathbb{P}_{s}^{1}$ is a multisection of the elliptic fibration $Y \rightarrow \mathbb{P}^{1}$. We want to find an elliptic saliently ramified multisection among the $D_{s}$. Then we apply 2.11 .

Denote by $R^{0} \subset R$ the Zariski open subset of points where the curve $R$ is smooth and where it is not tangent to the generators of the cone $Q$. For every point $P \in R^{0}$ we consider the affine line $L_{P}$ of sections $\mathbb{P}_{s}^{1}$ which are tangent to $R$ at $P$. These sections cover the whole cone $Q$, except the line joining $P$ and the vertex of $Q$. Consider the subset of sections of $\mathbb{P}_{\mathbb{P}^{1}}(\mathcal{O}(2))$ which have at least two distinct points of local intersection multiplicity $\geq 2$ with $R$. If this subset covers birationally the cone $Q$, then we obtain a 1-dimensional family of elliptic curves which covers $Y$ and which is generically transversal to the elliptic fibration $Y \rightarrow \mathbb{P}^{1}$. Hence we can apply 2.13 .

In particular, if the curve $R$ has a singular double point $r \in R$, then for any $P \in R^{0}$ we can find a tangent section $\mathbb{P}_{s}^{1}$ which passes through $r$. The family of such sections covers $Q$ (birationally), provided that $R$ has a component which is not a section. Therefore, we have only to consider the case when $R$ consists of 4 distinct components which are sections. Suppose that $r$ lies on the intersection of two components. Then the tangents to some point of a third component passing through $r$ cover $Q$ birationally and the argument above applies.

The only remaining case is the case of a non-singular curve $R$. For any $P \in R$ there exists a section $s \in L_{P}$ which has local intersection multiplicity $\geq 2$ with $R$ at some other point. Indeed, we have a natural tangent correspondence $T C_{P} \subset L_{P} \times R$ defined by

$$
T C_{P}=\left\{(s, r) \mid s \in L_{P}, r \in \mathbb{P}_{s}^{1} \cap R\right\} .
$$

Since $R$ is irreducible this correspondence is irreducible. Since $R$ is not a rational curve this correspondence has ramification points over $L_{P}$. Ramification points correspond to sections having at least two intersection points of local multiplicity $\geq 2$ with $R$. Hence, the family of sections with this property (as $P$ moves over $R^{0}$ ) covers (birationally) the cone $Q$ and defines a transversal elliptic fibration on $Y \rightarrow \mathbb{P}^{1}$.

Acknowledgements. The second author is very grateful to Barry Mazur and Joe Harris for their ideas and suggestions. We would like to thank I. Dolgachev for his remarks. The paper was written while both authors were enjoying the hospitality of the Max-Planck Institute in Bonn. The first author was partially supported by the NSF. The second author was partially supported by the NSA. We would like to thank the referee for the detailed comments which helped us to improve the exposition. 


\section{References}

[1] W. Barth and C. Peters, Automorphisms of Enriques surfaces, Invent. Math. 73 (1983), $383-411$.

[2] W. Barth, C. Peters and A. van de Ven, Compact complex surfaces, Ergebnisse der Mathematik und ihrer Grenzgebiete (3) [Results in Mathematics and Related Areas (3)], 4, Springer-Verlag, Berlin-New York, 1984.

[3] F. A. Bogomolov and Yu. Tschinkel, On the density of rational points on elliptic fibrations, Preprint 1998.

[4] F. Cossec and I. Dolgachev, Enriques surfaces I, Progress in Mathematics, 76. Birkhäuser Boston, Inc., Boston, MA, 1989.

[5] I. Dolgachev, On automorphisms of Enriques surfaces, Invent. Math. 76 (1984), 163-177.

[6] J. Harris and Yu. Tschinkel, Rational points on quartics, Preprint 1998.

[7] V. A. Iskovskikh and I. R. Shafarevich, Algebraic surfaces. Algebraic geometry, II, 127-162, Encyclopaedia Math. Sci., 35, Springer, Berlin, 1996.

[8] S. Kondo, Enriques surfaces with finite automorphism groups, Japan. J. Math. (N.S.) 12 (1986), 191-282.

[9] L. Merel, Bornes pour la torsion des courbes elliptiques sur les corps de nombres, Invent. Math. 124 (1996), 437-449.

[10] V. Nikulin, Description of automorphism groups of Enriques surfaces, Dokl. Akad. Nauk SSSR 277 (1984), 1324-1327.

[11] J. Silverman, Heights and the specialization map for families of abelian varieties, J. Reine Angew. Math. 342 (1983), 197-211.

Courant Institute of Mathematical Sciences, N.Y.U., 251 Mercer St., New York, NY 10012

E-mail address: bogomolo@cims.nyu.edu

Department of Mathematics, University of Illinois, Chicago, 851 South Morgan St., Chicago, IL 60607

E-mail address: yuri@math.uic.edu 